\title{
Maintien d'un allèle délétère de stérilité mâle dans certaines populations naturelles du crustacé marin Jaera albifrons
}

\author{
P. LÉCHER * et Marie-Louise CARIOU ** \\ * Laboratoire de Génétique et Biologie Cellulaire, Université de Clermont-Ferrand 2, \\ BP 45, 63170 Aubière, France
}

** Centre National de la Recherche Scientifique, Laboratoire de Biologie et Génétique Evolutives, 91198 Gif-sur-Yvette Cedex, France

\begin{abstract}
Résumé
Dans les populations de la Côte basque de Jaera (a.) albifrons, certains mâles présentent une malformation de l'appareil copulateur qui entraîne une stérilité mécanique, la spermatogenèse étant normale. Cette anomalie dépend d'un allèle autosomal récessif. La fréquence des mâles anormaux dans ces populations est en moyenne de $5,8 \%$, ce qui correspond à une fréquence allélique de 0,24 . Les mâles homozygotes récessifs n'ayant pas de descendants, le gène devrait être progressivement éliminé des populations. Le maintien d'un allèle désavantageux, à une fréquence élevée, dans les populations naturelles, fait l'objet de plusieurs hypothèses.
\end{abstract}

Mots clés : allèle délétère, population, stérilité mâle, Jaera.

\section{Summary}

Persistence of a deleterious allele for male sterility in some natural populations of the marine crustacea Jaera albifrons

A morphological anomaly of the copulative system of males of the species Jaera (a.) albifrons was detected in the populations of the Côte Basque. These males have normal spermatogenesis but are not able to mate. This anomaly is governed by an autosomal recessive allele. In these populations, the frequency of abnormal males is at an average of $5.8 \%$. The allele frequency is 0.24 . Homozygous recessive males do not produce progeny and thus elimination of the gene was to be expected. Several hypotheses concerning the persistence of this deleterious allele at a relatively high frequency in natural populations are discussed.

Key words : deleterious allele, population, male sterility, Jaera.

\section{Introduction}

Les études génétiques et cytogénétiques (LÉCHER, 1968) menées sur Jaera albifrons ayant conduit à l'analyse de nombreuses populations, nous avons observé dans les plus 
méridionales d'entre elles des individus mâles qui présentent une malformation de l'appareil copulateur (Bocquet \& LÉcher, 1968). Chez le mâle de Jaera, l'appareil copulateur est constitué des deux premières paires de pléopodes (BocQueT, 1953) : la première paire (droit et gauche fusionnés) forme le préopercule; la deuxième est constituée par les stylets copulateurs qui coulissent à l'intérieur du préopercule. Chez les mâles anormaux, qui présentent par ailleurs une spermatogenèse normale, des vésicules séminales remplies de spermatozoïdes et des caractères sexuels secondaires normaux, les stylets copulateurs existent mais le préopercule est absent. Ces individus sont incapables de féconder les femelles.

Des croisements effectués au laboratoire ont permis de conclure que cette anomalie est déterminée par un gène autosomal récessif (BocQueT \& LÉCHER, 1968). Trois génotypes existent dans l'un et l'autre sexe : $A A, A a$, $a a$; les mâles $a a$ qui ne possèdent pas de propercule ne peuvent transmettre le caractère. Bien que cette anomalie entraîne une stérilité mécanique, elle persiste aussi bien en élevage que dans les populations naturelles où il a paru intéressant d'étudier sa fréquence.

\section{Matériel et méthodes}

La localisation géographique des stations de la Côte basque où ont été observés des mâles anormaux est indiquée sur la figure 1. Toutes ces populations correspondent à la limite sud de distribution de l'espèce. Dans cette région, les stations où des Jaera ont été trouvées sont rares, éloignées les unes des autres et d'effectifs faibles puisque les échantillons étudiés varient de 144 à 437 individus.

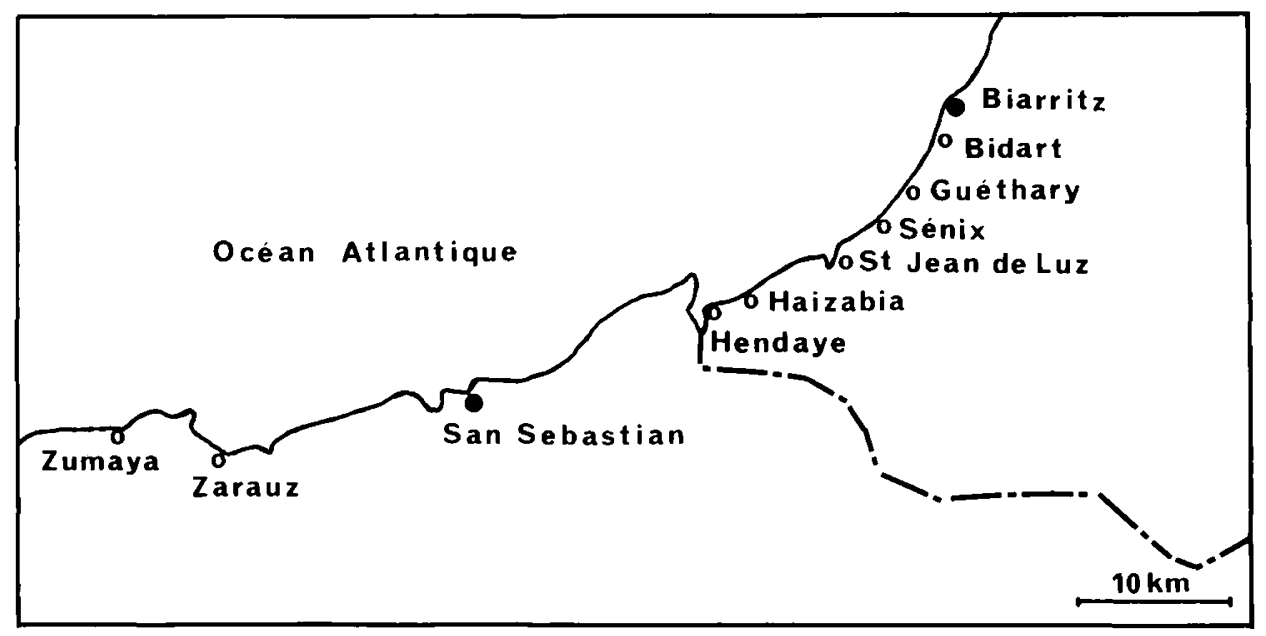

FIG. 1

Localisation des stations de Jaera (a.) albifrons sur la Côte Basque.

Map showing the locations of populations of Jaera (a.) albifrons used in this study. 
Plus au nord, l'espèce albifrons devient beaucoup plus abondante ; il est souvent possible, dans une même station, de récolter plusieurs milliers d'individus parmi lesquels des mâles porteurs de l'anomalie du préopercule n'ont jamais été observés.

L'identification des mâles anormaux, donc stériles, a été faite par simple examen au microscope binoculaire. L'examen des échantillons de Jaera adultes a aussi permis de déterminer les proportions respectives des mâles et des femelles.

\section{Résultats}

Les résultats observés dans huit populations de la Côte basque sont rassemblés dans le tableau 1.

\section{TABLEAU 1}

Caractéristiques des populations de la Côte basque où des mâles porteurs d'une anomalie ont été observés. (n : nombre d'individus, $\mathrm{p}$ : pourcentage)

Characteristics of the Jaera (a.) albifrons populations where a morphological anomaly of the copulative system of males was detected ( $\mathrm{n}:$ number of abnormal males, $\mathrm{p}:$ percentage)

\begin{tabular}{l|c|c|c|c|c}
\hline \multirow{2}{*}{ Populations } & \multirow{2}{*}{$\begin{array}{c}\text { Effectif } \\
\text { total }\end{array}$} & Mâles & $\begin{array}{c}\text { Proportion } \\
\text { de mâles (\%) }\end{array}$ & \multicolumn{2}{|c}{ Mâles anormaux } \\
\cline { 5 - 6 } & & & & $n$ & $p \%$ \\
\hline Bidart & 147 & 55 & 37,4 & 3 & 5,45 \\
Guéthary & 184 & 82 & 44,5 & 4 & 4,88 \\
Senix & 224 & 88 & 39,3 & 4 & 4,55 \\
Saint-Jean-de-Luz & 153 & 64 & 41,8 & 2 & 3,12 \\
Haïzabia (1966) & 414 & 154 & 37,2 & 8 & 5,19 \\
Haïzabia (1982) & 437 & 162 & 37,1 & 9 & 5,56 \\
Hendaye & 193 & 67 & 34,7 & 5 & 7,46 \\
Zarauz & 185 & 80 & 43,2 & 5 & 6,25 \\
Zumaya & 144 & 64 & 44,4 & 7 & 10,94 \\
\hline Totaux & 2081 & 816 & 39,2 & 47 & 5,76 \\
\hline \hline
\end{tabular}

Ces populations se caractérisent par leur grande homogénéité. La proportion des mâles est régulièrement plus faible que celle des femelles, environ 1 mâle pour 2 femelles. Il s'agit d'une observation habituelle dans les populations naturelles de Jaera albifrons, alors qu'à la naissance au laboratoire, il y a autant de mâles que de femelles (Cléret, 1966). Le résultat paraît dû à une moins grande longévité du sexe mâle (Veuille, 1982), mais aussi sans doute à un moins bon échantillonnage des mâles dans les populations naturelles en raison de leur plus petite taille.

Toutes les populations basques ont fourni une faible proportion (de 3 à $11 \%$ ) de mâles anormaux, donc stériles. Compte tenu des effectifs disponibles, les différences ne 
sont pas significatives ( $\chi^{2}$ d'homogénéité $=4,81$ pour $8 \mathrm{ddl}$ ). Nous pouvons donc considérer que les populations ont une structure génétique identique qui, de plus, semble se maintenir au cours du temps : la station de Haïzabia, prospectée à 16 ans d'intervalle, a fourni des résultats remarquablement stables.

Comme cela a été démontré (BocQuet \& LéchER, 1968), l'allèle $a$, responsable de l'anomalie est un allèle récessif qui n'interfère pas avec le déterminisme du sexe mais dont l'expression est limitée au sexe mâle. Dans le but d'estimer l'ordre de grandeur de la fréquence de l'allèle $a$ dans les populations naturelles, on peut considérer, en première approximation, que les fréquences $\mathrm{p}$ et $\mathrm{q}$ des allèles $A$ et $a$ sont identiques dans les deux sexes et que les femelles $a a$, non détectées, existent dans les mêmes proportions que les mâles $a a$ "stériles". Dans ces conditions, la fréquence 0,058 des mâles anormaux correspond à $\mathrm{q}^{2}$ et l'on déduit que la fréquence de l'allèle $a$ est de l'ordre de 0,24 .

En réalité, compte tenu du fait que les mâles porteurs de l'allèle délétère $a$ à l'état homozygote ne contribuent pas à la génération suivante, les structures gamétiques diffèrent dans les deux sexes. On supposera que les populations naturelles sont panmictiques bien que l'on ne puisse exclure une consanguinité de position (CARIOU \& LÉCHER, en préparation) chez ces animaux sédentaires qui ne possèdent pas de phase libre au cours de leur développement. Dans ces conditions, si on note $q_{n}^{\prime}$ et $q_{n}^{\prime \prime}$ les fréquences gamétiques de l'allèle délétère $a$ respectivement chez les mâles et chez les femelles de la génération $G_{n}$, les gamètes femelles et mâles se recombinant au hasard, la structure génotypique est la même dans les deux sexes d'une même génération. Ainsi, dans la génération $\mathrm{G}_{\mathrm{n}+1}$, elle est la suivante :

- fréquence du génotype $A A=\left(1-\mathrm{q}_{\mathrm{n}}^{\prime}\right)\left(1-\mathrm{q}^{\prime \prime}\right)$

- fréquence du génotype $A a=\mathrm{q}_{\mathrm{n}}^{\prime}\left(1-\mathrm{q}_{\mathrm{n}}^{\prime \prime}\right)+\mathrm{q}_{\mathrm{n}}^{\prime \prime}\left(1-\mathrm{q}_{\mathrm{n}}^{\prime}\right)$

- fréquence du génotype $a a=\mathrm{q}^{\prime}{ }_{n} \mathrm{q}^{\prime \prime}{ }_{\mathrm{n}}$

En revanche, les structures gamétiques diffèrent dans les deux sexes. On montre que chez les femelles, sous l'hypothèse d'une valeur sélective identique des différents génotypes, la fréquence gamétique de l'allèle $a$ à la génération $\mathrm{G}_{\mathrm{n}+1}$ est égale à la moyenne arithmétique des fréquences gamétiques chez les mâles et chez les femelles de la génération précédente $\mathrm{G}_{\mathrm{n}}$ (c'est-à-dire à la fréquence globale de $a$ chez les individus de $\left.G_{n+1}\right)$.

$$
\mathrm{q}^{\prime \prime}{ }_{\mathrm{n}+1}=\left(\mathrm{q}_{\mathrm{n}}^{\prime}+\mathrm{q}_{\mathrm{n}}^{\prime \prime}\right) / 2
$$

Chez les mâles, seuls les génotypes $A A$ et $A a$ contribuent au pool gamétique de sorte que :

$$
\mathrm{q}_{n+1}^{\prime}=\frac{\left(\mathrm{q}_{\mathrm{n}}^{\prime}+\mathrm{q}_{\mathrm{n}}^{\prime \prime}\right) / 2-\mathrm{q}_{\mathrm{n}}^{\prime} \mathrm{q}_{\mathrm{n}}^{\prime \prime}}{1-\mathrm{q}_{\mathrm{n}}^{\prime} \mathrm{q}_{\mathrm{n}}^{\prime \prime}}
$$

La fréquence gamétique de l'allèle $a$ chez les mâles est systématiquement plus faible que chez les femelles tant que l'équilibre, où $\mathrm{q}^{\prime}=\mathrm{q}^{\prime \prime}=0$, n'est pas atteint.

Ainsi, l'évolution des structures génotypiques et gamétiques au fil des générations selon le modèle génétique considéré entraîne une décroissance régulière de la fréquence de l'allèle $a$. 


\section{Discussion et conclusion}

La décroissance théorique de l'allèle $a$, responsable de l'anomalie du préopercule des mâles de Jaera albifrons, dans une population panmictique est moins rapide que celle d'un allèle récessif létal dans les deux sexes. Elle devrait cependant conduire à l'élimination du gène défavorable, même si la convergence vers $q=0$ est lente puisque les mâles homozygotes $a a$ deviennent de plus en plus rares dans la population. Tout au plus, l'allèle $a$ ne devrait exister dans les populations naturelles qu'à une très faible fréquence en équilibre avec le taux de mutation spontanée. Les observations régulières de 1959 à 1984 (LÉCHER, non publié) montrent clairement que ceci n'est pas le cas et que l'allèle $a$ est toujours présent et se maintient à une fréquence très élevée (environ $20 \%$ ) pour un allèle délétère. L'argument le plus convaincant est fourni par la stabilité de la fréquence des mâles anormaux dans la population d'Haïzabia à plus d'une cinquantaine de générations d'intervalle. Cette situation rappelle celle rapportée par GÉNERMONT (1981). Deux catégories d'hypothèses peuvent expliquer une telle observation.

Une première catégorie implique des processus sélectifs, c'est-à-dire un succès reproductif différent selon le génotype. Pour compenser l'élimination sélective des mâles $a a$, on peut envisager un avantage sélectif des femelles $a a$ ou des hétérozygotes.

Une seconde catégorie d'hypothèses met en jeu des processus stochastiques qui sont eux-mêmes de deux sortes. On peut en premier lieu imaginer que l'équilibre observé est maintenu par des mutations spontanées de $A$ vers $a$. Le taux de mutation est inconnu chez Jaera, mais en supposant qu'il soit du même ordre de grandeur que celui des insectes $\left(1 \cdot 10^{-6}\right)$ ou même que celui des mammifères $\left(1 \cdot 10^{-4}\right)$, ceci reste très invraisemblable puisqu'il faudrait un taux supérieur à $2 \%$ pour compenser la perte régulière de $a$ à chaque génération. Un second mécanisme est de faire intervenir une distorsion de ségrégation qui, chez les hétérozygotes, favoriserait la production de gamètes $a$ au détriment de $A$. Des phénomènes de ce genre sont connus chez diverses espèces et l'on sait par exemple qu'ils permettent, chez les souris, le maintien d'allèles délétères au locus T (BENNETT, 1975).

Un choix entre ces diverses hypothèses impliquerait des expériences extensives en laboratoire, ce qui n'est guère envisageable compte tenu des difficultés d'élevage des Jaera. En l'état actuel de nos connaissances, on peut supposer que la mutation $a$ est apparue dans les populations basques où, malgré la discontinuité des peuplements actuels, elle aurait diffusé et où elle serait maintenue par l'un ou plusieurs des processus envisagés ci-dessus.

Son absence dans les populations plus nordiques relèverait simplement d'un phénomène historique, c'est-à-dire l'absence de flux génique lié à l'absence des Jaera le long des côtes sableuses des Landes.

Reçu le 10 août 1987.

Accepté le 16 octobre 1987. 


\section{Remerciements}

Les auteurs remercient Ph. L'Héritier, J. Génermont de leurs critiques et conseils et J.R. David de ses suggestions qui ont sensiblement amélioré le manuscrit.

\section{Références bibliographiques}

Bennett D., 1975. The T locus of the mouse. Cell., 6, 441-454.

BocQuet C., 1953. Recherches sur le polymorphisme naturel des Jaera marina (Isopodes, Asellotes). Arch. Zool. Exp. Gen., 90, 187-450.

Bocquet C., LÉcher P., 1968. Anomalie génétique entraînant la stérilité chez certains mâles de Jaera albifrons (Crustacé, Isopode). C.R. Soc. Biol., 162, 355-358.

Cléret J.J., 1966. Le rapport numérique des sexes dans quelques populations naturelles de Jaera albifrons Leach. Bull. Soc. Linn. Normandie, série 10, 7, 155-161.

GÉnermont J., 1981. Maintien d'un gène létal dans une population : le cas des chats sans queue de l'île de Man. Ann. Génét. Sél. Anim., 13, 371-380.

LÉCHER P., 1968. Cytogénétique de l'hybridation expérimentale et naturelle chez l'Isopode Jaera albifrons. Arch. Zool. Exp. Gén., 108, 633-698.

Veuille M., 1982. L'évolution des systèmes de reproduction, du dimorphisme sexuel et du comportement sexuel chez Jaera (Isopode, Asellote). Thèse de Doctorat ès Sciences, Paris VI. 